Results: CT revealed erosions and ankyloses of the sacroiliac joints, fusion of the posterior elements of vertebra especially in the thoracic spine, enthesophytes, not seen with CRs, in 26 patients $(p<0,05)$.

Level of diagnostic confidence was higher when interpreting CT compared to CRs, in cases of erosions and ankyloses, in 35 patients $(p<0,05)$.CT was slightly superior in the detection and characterization of syndesmophytes, enthesopathy and calcifications.

Effective dose for "i-Dose 5" CT was $1.20 \pm 0.26 \mathrm{mSv}$ and for CR $=1.07 \pm 0.21$ $\mathrm{mSv}$. The difference was not statistically significant $(p=0.588)$.

Conclusion: iDose CT of spine and pelvis at an effective dose, lower than previously published, seems to perform better than radiographs in the detection of structural changes of PsSpA. Further studies are needed to document the capabilities of iDose CT for the imaging assessment of PsSpA in daily clinical practice.

References:

[1] Baraliakos X, et al (2009)The Natural Course of Radiographic Progression in Ankylosing Spondylitis - Evidence for Major Individual Variations in a Large Proportion of Patients The Journal of Rheumatology May, 36 (5) 997-1002

[2] de Koning A et, al (2018) Low-dose CT detects more progression of bone formation in comparison to conventional radiography in patients with ankylosing spondylitis: results from the SIAS cohort. Ann Rheum Dis.;77(2):293-299.

Disclosure of Interests: None declared

DOI: 10.1136/annrheumdis-2020-eular.6631

\section{SAT0551 \\ WHOLE BODY MACROPHAGE PET IMAGING THAT INCLUDES THE FEET CAN PROVIDE ADDITIONAL INFORMATION TO CLINICAL ASSESSMENT IN PATIENTS WITH EARLY RHEUMATOID ARTHRITIS}

N. J. F. Verweij ${ }^{1}$, M. Ter Wee ${ }^{1,2}$, J. De Jongh ${ }^{1}$, G. C. J. Zwezerijnen ${ }^{3}$, M. Yaqub ${ }^{3}$, M. Boers ${ }^{1,2}$, A. Voskuyl ${ }^{1}$, A. A. Lammertsma ${ }^{3}$, W. Lems ${ }^{1}$, C. J. Van der Laken ${ }^{1}$. ${ }^{1}$ Amsterdam Rheumatology and Immunology Center, Amsterdam UMC - location VUmc, Department of Rheumatology, Amsterdam, Netherlands; ${ }^{2}$ Amsterdam Public Health, Amsterdam UMC - Location VUmc, Department of Epidemiology and Biostatistics, Amsterdam, Netherlands; ${ }^{3}$ Amsterdam UMC - location VUmc, Department of Radiology \& Nuclear Medicine, Amsterdam, Netherlands

Background: Clinical assessment of arthritis is the cornerstone in the diagnosis and treatment of rheumatoid arthritis (RA). Nevertheless, reliable determination of (sub)clinical arthritis can be difficult, especially in the feet. Advanced imaging techniques may contribute to early diagnosis and therapy monitoring through sensitive detection and (quantitative) monitoring of synovitis. Previously, it has been demonstrated that macrophage imaging using $(R)-\left[{ }^{11} \mathrm{C}\right] \mathrm{PK} 11195$ positron emission tomography (PET) allows for highly sensitive and specific imaging of RA disease activity in the hands. ${ }^{(1,2)}$ Whole body macrophage PET imaging that includes the feet has not yet been evaluated in RA.

Objectives: To compare whole body macrophage PET imaging to clinical assessment of arthritis activity in clinically active, early RA patients.

Methods: Thirty-five previously untreated RA patients (age $54 \pm 12,51 \%$ male) with at least two clinically inflamed joints were included. They underwent a whole body $(R)-\left[{ }^{11} \mathrm{C}\right] \mathrm{PK} 11195 \mathrm{PET} / \mathrm{computed}$ tomography (CT) scan in addition to standard clinical assessment of number of tender and swollen joints (TJC and SJC, respectively). Two readers blinded to clinical assessment ( $G Z$ and $C v d L)$ visually scored intensity of uptake in joints on a 0 to 3 scale. A PET positive joint score was defined at $\geq 1$. Additionally, $(R)-\left[{ }^{11} \mathrm{C}\right] \mathrm{PK} 11195$ uptake in joints was assessed quantitatively as standardized uptake values (SUV). Visual parameters were compared to clinical parameters using Cohen's kappa, and quantitative parameters were analyzed using an independent T-test.

Results: All patients showed enhanced tracer uptake in one or more joints (Figure 1). A total of 168 joints were visually PET positive, with the following distribution: $16 \%$ in the wrists, $14 \%$ in the metacarpophalangeal joints, $25 \%$ in the proximal interphalangeal joints, $4 \%$ in the ankles, $37 \%$ in the metatarsophalangeal joints. Positivity in other large joints was rare (4\%). The number of discrepant findings between PET and clinical outcome (TJC and/or SJC) varied based on anatomic localization; more joints were clinically active in the hands, and more joints were active on the PET scan in the feet. Consequently, agreement between visual PET positivity and clinical activity was low, with only moderate agreement found in the ankles ( $\kappa=0.46$ and 0.41 for SJC and TJC respectively). Quantitative PET data showed a trend towards higher SUV values in joints that were clinically tender and/or swollen, reaching a significant difference in the feet (ankles + MTPs) versus SJC (Figure 2; 0.7 vs 1.0, $p<0.001$ ). However, parts of the clinically non-affected joints also depicted moderately increased SUV values, and vice versa.

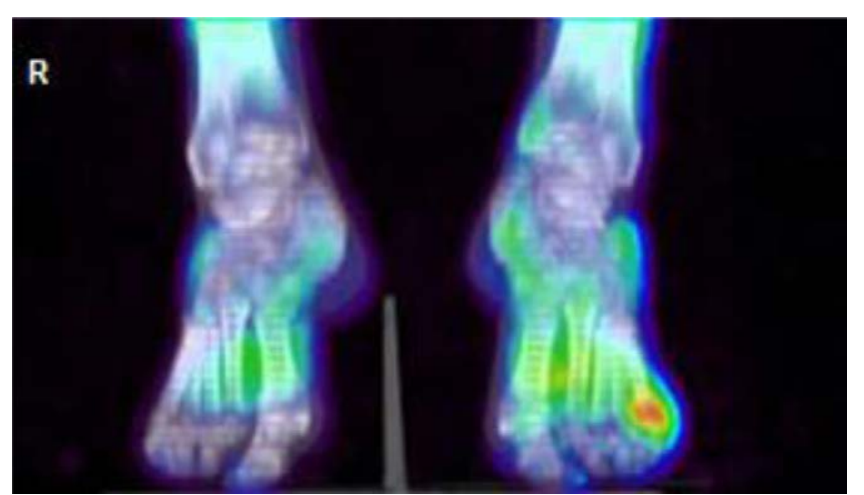

Figure 1. Visual PET uptake in the left MTP5-joint

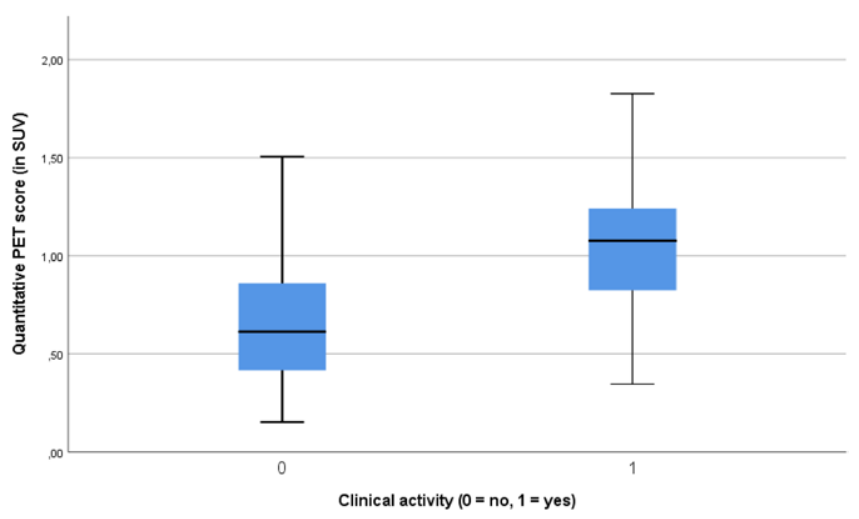

Figure 2. (R)-[11C]PK11195 (SUV) in both clinically affected and non-affected feet joints (defined as swollen yes or no).

Conclusion: Whole body macrophage PET imaging showed clear uptake of $(R)$ $\left[{ }^{11} \mathrm{C}\right] \mathrm{PK} 11195$ in several joints of clinically active, early RA patients, especially in MTP-joints. The best correlation between quantitative PET data and clinical assessment of swelling was observed in the feet. In general, however, PET also provided distinct information from clinical assessment, which may provide a means for detecting subclinical synovitis. We are performing longitudinal studies to further assess the value of macrophage PET in RA.

References:

[1] Elzinga EH, et al. J Nucl Med. 2011; 52(1): 77-80.

[2] Gent YY, et al. J Rheumatology. 2014; 41: 2145-52

Acknowledgments: We thank ReumaNederland and Pfizer for financial support of this investigator initiated study.

Disclosure of Interests: Nicki J.F. Verweij: None declared, Marieke ter Wee: None declared, Jerney de Jongh: None declared, Gerben C.J. Zwezerijnen: None declared, Maqsood Yaqub: None declared, Maarten Boers: None declared, Alexandre Voskuyl: None declared, Adriaan A. Lammertsma: None declared, WIIlem Lems Grant/research support from: Pfizer, Consultant of: Lilly, Pfizer, Conny J. van der Laken: None declared

DOI: 10.1136/annrheumdis-2020-eular.4380

\section{SAT0552 IMAGING OF CORTICAL INTERRUPTION IN THE METACARPOPHALANGEAL JOINTS WITH HIGH- RESOLUTION PERIPHERAL QUANTITATIVE COMPUTED TOMOGRAPHY SHOWS HIGH RELIABILITY AND ACCEPTABILITY FOR PATIENTS WITH RHEUMATOID ARTHRITIS.}

R. Klose-Jensen ${ }^{1,2}$, K. K. Keller ${ }^{3}$, B. Langdah ${ }^{4}$, E. M. Hauge ${ }^{1,2} .{ }^{1}$ Aarhus University Hospital, Department of Rheumatology, Aarhus N, Denmark; ${ }^{2}$ Aarhus University, Department of Clinical Medicine, Aarhus N, Denmark; ${ }^{3}$ Silkeborg Regional Hospital, Diagnostic Centre, Silkeborg, Denmark; ${ }^{4}$ Aarhus University Hospital, Department of Endocrinology and Internal Medicine, Aarhus N, Denmark

Background: High-Resolution peripheral Quantitative Computed Tomography (HR-pQCT) has been proposed as a modality for assessment of disease severity in inflammatory arthritis. The excellent resolution $\left(82 \mu \mathrm{m}^{3}\right)$ and the inherent contrast between bone and soft tissue makes it a gold standard reference for the detection of bone damage in vivo. However, the nine minutes acquisition 
time for the metacarpophalangeal (MCP) joints might yield some disadvantages. Having the hand and arm immobilised for this long might discomfort the patients, thereby reducing acceptability, resulting in poor adherence. The longer imaging time might also increase the risk of motion-induced image degradation.

Objectives: The objective of this study was two-fold. Firstly, we investigated motion-induced image degradation of 2nd and 3rd MCP joints for two methods of standardised positioning of the hand. Secondly, the acceptability of HR-pQCT imaging was explored for patients with established Rheumatoid Arthritis (RA). Methods: Fifty patients with RA had their 2nd and 3rd MCP joints imaged by HR-pQCT. The patients were scanned two times, using a custom-made positioning splint, with and without an inflatable immobilisation device. In order to investigate acceptability, the patients were afterwards given a questionnaire regarding their procedure experience of HR-pQCT imaging with and without the inflatable hand immobilisation device. For each acquisition, the image quality was graded, and the number, width, depth and length of cortical interruptions were measured. Twenty percent of the acquisitions were reevaluated to determined intrareader reliability using the intraclass correlation coefficient (ICC).

Results: The acceptability regarding HR-pQCT imaging was high, with only $6 \%$ preferring conventional X-ray compared to $40 \%$ of the patients preferring HR-pQCT imaging. The remaining 54\% were indifferent to the modality. Seventy-four percent found it hard to keep their fingers at rest during the imaging. Fifty percent of the patients thought the inflatable hand immobilisation device helped keep their fingers at rest compared to only $6 \%$ who believed it impaired their ability to keep their fingers at rest. This was not observable in the image quality, however, as the overall image quality was high and no clinically relevant difference of the visual grading between the acquisitions with and without the inflatable hand immobilisation device was observed. The number, width, depth and length of cortical interruption all indicated excellent reproducibility as shown in table 1. No discernible difference between the two acquisitions was observed.

Table 1. Intraclass correlation coefficients for the number, width, depth and length of cortical interruptions, with and without the inflatable hand immobilization device.

\begin{tabular}{lll}
\hline & Acquisition 1 & Acquisition 2 \\
\hline Cortical interruptions number & $0.99(0.94$ to 1.00$)$ & $0.98(0.91$ to 1.00$)$ \\
Average cortical interruption width & $0.98(0.92$ to 0.99$)$ & $0.99(0.95$ to 1.00$)$ \\
Average cortical interruption depth & $0.98(0.92$ to 0.99$)$ & $0.97(0.89$ to 0.99$)$ \\
Average cortical interruption length & $0.93(0.75$ to 0.98$)$ & $0.98(0.94$ to 1.00$)$
\end{tabular}

Acquisition 1 - Without the inflatable hand immobilization device.

Acquisition 2 - With the inflatable hand immobilization device.

Data presented as mean ( $95 \%$ confidence intervals).

Conclusion: The high acceptability signifies the feasibility of the novel HR-pQCT imaging; this was evident by the fact that more patients preferred HR-pQCT imaging compared to conventional X-ray examination. The inflatable hand immobilisation device did not reduce motion-induced image degradation as the overall image quality was high for imaging of the MCP joints in both acquisitions. Our result, however, shows that the patients are more than capable of keeping their fingers at rest for the long acquisition time.

Disclosure of Interests: Rasmus Klose-Jensen: None declared, Kresten Krarup Keller: None declared, Bente Langdahl Grant/research support from: Amgen, NovoNordisk, Consultant of: Amgen Inc., Eli Lilly, UCB Pharma, Ellen Margrethe Hauge: None declared

DOI: 10.1136/annrheumdis-2020-eular.3728

\section{SAT0553 QUANTITATIVE ANALYSIS OF IMAGING FEATURES AT CHEST CT OF PULMONARY ARTERIAL AND VENOUS COMPONENTS IN SYSTEMIC SCLEROSIS- INTERSTITIAL LUNG DISEASE (SSC-ILD).}

C. Bruni ${ }^{1}$, M. Occhipinti ${ }^{2,3}$, G. Camiciottoli ${ }^{3}$, M. Bartolucci ${ }^{4}$, M. Pienn ${ }^{5}$, G. Lepri ${ }^{1}$,

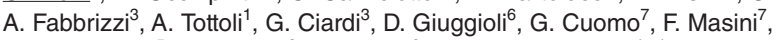
H. Olschewski ${ }^{5}$, F. Lavorini ${ }^{3}$, L. Calistri ${ }^{2}$, M. Matucci-Cerinic ${ }^{1}{ }^{1}{ }^{1}$ University of Florence, Rheumatology, Firenze, Italy; ${ }^{2}$ University of Florence, Radiology, Florence, Italy; ${ }^{3}$ University of Florence, Pulmonology, 50141, Italy; ${ }^{4}$ Careggi University Hospital, Radiology, Firenze, Italy; ${ }^{5}$ Ludwig Boltzmann Institute for Lung Vascular Research, Institute of Physiology, Graz, Austria; ${ }^{6}$ Policlinico of Modena University Hospital of Modena, Rheumatology, Modena, Italy; ${ }^{7}$ University of Campania Luigi Vanvitelli, Internal Medicine, Naples, Italy

Background: Interstitial lung disease (ILD) and pulmonary arterial hypertension carry a negative impact on SSc prognosis. Chest CT is the gold standard in assessing ILD and helps in evaluating associated vascular involvement.

Objectives: As qualitative analysis of CT scans is limited by low reproducibility and time constraints, we aimed at evaluating parenchymal and vascular features in SSc-ILD by quantitative analysis (QA) of CT scans and testing the relationship with clinical-functional data.
Methods: We prospectively enrolled 80 patients who underwent PFTs and chest CT scan spirometry gated at TLC on the same day. Clinical, lung functional and diffusion data, as well as disability indexes were collected. CT images were analyzed by a computational platform for texture analysis of ILD patterns (CALIPER), through Imbio LTA. It quantified the extent of normal pattern (NP \%), ground glass opacities (GG\%), reticulation (RET \%), honeycombing (HC \%), total ILD extent (ILD EXT \%) and hyperlucent (HL \%). Low density areas, representing emphysematous area, were also quantified (LDA \%). For lung vessel analysis, a software program developed by the Ludwig Boltzmann Institute for Lung Vascular Research was used. This software determined total, arterial, and venous vascular volumes (TV, AV, VV), and relative volumes (TV\%, AV\%, VV\%), as well as density and number for total, arterial and venous vessels.

Results: $43 / 80$ patients/CT scans were eligible for both software analyses, while $36 / 43$ for arterial and venous separation. TV\% and total vessel density were correlated positively with $\mathrm{mRSS}$ and negatively with \%FVC ( $\mathrm{r}=-0.537$ and $\mathrm{r}=-0.382)$ and \%TLC ( $\mathrm{r}=$ 0.511 and $r=-0.648$ ), while vessel tortuosity correlated positively with \%DLco. This was confirmed when separately analyzing arterial vessels, while VV\% negatively correlated with \%FVC, \%TLC and \%DLco. There was a positive correlation between \%ILD patterns and \%vascular volumes, being significant for TV\%-AV\%, total vessels and arterial density. Conversely, \%ILD patterns were negatively correlated with VV and number of veins detected, despite positive correlation between VV\% and ILD_EXT\%. When clustering patients according to \%FVC and \%DLco with $80 \%$ normal cutoff, \%FVC allowed clustering according to significantly different ILD patterns extents and vascular features, while \%DLCO for vascular features only. Moreover, the consecutive addition of functional impairment and worsening of ILD (from both normal \%FVC and \%Dlco, to \%DLco impairment only to both \%FVC and \%Dlco impairment), there was a significant increase in \%TV, \% AV and \%VV, with the exception of decrease in \%VV and venous density in patients with double impairment versus DLco single impairment.

Conclusion: This is the first study showing in SSc a direct correlation between ILD and the increase in lung vascular volume, which is characterized by increase in arterial volume and density and reduction in venous volume and number These results might be explained by the reduction of pulmonary volume due to fibrosis. However, also a para-physiological mechanism of redistribution of blood flow in lung areas, less involved by ILD, might be considered. Further studies on lung vessel quantification and distribution are ongoing.

Disclosure of Interests: Cosimo Bruni Speakers bureau: Actelion, Eli Lilly, Mariaelena Occhipinti Consultant of: Imbio, Gianna Camiciottoli: None declared Maurizio Bartolucci: None declared, Michael Pienn: None declared, Gemma Lepri: None declared, Alessio Fabbrizzi: None declared, Alessandra Tottoli: None declared, Giuglia Ciardi: None declared, Dilia Giuggioli: None declared, Giovanna CUOMO: None declared, Francesco Masini: None declared, Horst Olschewski: None declared, Federico Lavorini: None declared, Linda Calistri: None declared, Marco Matucci-Cerinic Grant/research support from: Actelion, MSD, Bristol-Myers Squibb, Speakers bureau: Acetelion, Lilly, Boehringer Ingelheim DOI: 10.1136/annrheumdis-2020-eular.2177

\section{SAT0554 SONOGRAPHIC ASSESSMENT OF CALCIUM PYROPHOSPHATE DEPOSITION DISEASE AT WRIST. A FOCUS ONTHE SCAPHO-LUNATE LIGAMENT.}

E. Cipolletta ${ }^{1}$, G. Smerilli ${ }^{1}$, R. Mashadi Mirza ${ }^{2}$, A. DI Matteo ${ }^{1,3}$, F. Salaffi $^{1}$, W. Grassi ${ }^{1}$, E. Filippucci ${ }^{1} .{ }^{1}$ Università Politecnica delle Marche, Rheumatology Unit, Jesi, Ancona, Italy; ${ }^{2}$ ULSS 6 Euganea, Dipartimento di Diagnostica per Immagini, Padova, Italy; ${ }^{3}$ University of Leeds, Leeds Institute of Rheumatic and Musculoskeletal Medicine, Leeds, United Kingdom

Background: Only few articles evaluated the wrist in calcium pyrophosphate deposition disease (CPPD), although it is the second most frequent target of CPPD. Very recently, in a computed tomography (CT) study ligamentous calcifications were reported as a highly specific feature of CPPD at wrist level (1).

Objectives: i) to determine the prevalence and distribution of the ultrasound (US) findings indicative of calcium pyrophosphate (CPP) crystal deposits at the wrist, with a particular focus on the dorsal aspect of the scapho-lunate ligament (SLL); ii) to investigate the diagnostic accuracy of US and conventional radiography (CR) in the evaluation of CPP crystal deposits at wrist level, iv) to assess the agreement between the different imaging techniques.

Methods: Consecutive patients with a "definite" diagnosis of CPPD according to the Ryan and McCarty criteria and disease controls were prospectively included in this cross-sectional single-centre study. Dorsal part of the SLL, triangular fibrocartilage complex (TFCC), and volar recess of the radio-lunate joint were explored using US (according to EULAR standard scans and OMERACT definitions), CR and CT.

Results: Sixty-one CPPD patients and 39 disease controls were enrolled. Two-hundred wrists were evaluated using both CR and US. CT data of $26(13.0 \%)$ wrists were available: 20 wrists in CPPD patients and 6 wrists in controls. CPP crystal deposits were found by US in at least one wrist in $95.1 \%$ of CPPD patients and in $15.4 \%$ of controls $(p<0.001)$. SLL calcification was reported in $83.6 \%$ of CPPD patients and in $5.1 \%$ of controls $(p<0.001)$. CPP crystal deposits were 